\title{
REDUCTION OF BLOOD PRODUCTS TRANSFUSIONS DURING LIVER TRANSPLANTATION
}

Luc Massicotte, M.D. *, Serge Lenis, M.D., †, Lynda Thibeault, MD MSc, André Roy, M.D., §

* Clinical Lecturer, $\dagger$ Director and Associate Professor, Anesthesiology Department of the Centre Hospitalier de l'Université de Montréal (CHUM)

$\S$ Associate professor, Department of surgery, Hepato-biliary Service, of the CHUM

\section{IMPLICATION STATEMENT}

This is a quasi experimental protocol: before-after study, comparing 61 liver transplantations conducted during a one year period to 206 transplantations (1998-2002) to evaluate red blood cells transfusion reduction strategies through central venous pressure reduction with complete avoidance of plasma transfusion, limitation of volume substitution and by using intraoperative phlebotomy.

Ba dkground: Correction of coagulation defects with plasma transfusion did not decrease the need for intraoperative red blood cells (RBC) transfusion during liver transplantations. On the contrary it has resulted in a hypervolemic state that results in an increase of shed blood. As well, plasma transfusion is associated with a decreased one-year survival rate. The aim of the present prospective survey was to evaluate whether anesthesiologists could reduce intraoperative RBC transfusion during liver transplantation by changing their anesthesia practice, more specifically by maintaining a low central venous pressure (CVP), by restricting volume replacement, by eliminating all plasma transfusion and by using intraoperative phlebotomy during the transplantation.

Methods: Sixty-one liver transplantations were prospectively studied during one-year period and were compared to a retrospective series (1998-2002). A low CVP was maintained in all patients prior the anhepatic phase. Coagulation disorders were not corrected pre-operatively, intraoperatively or post-operatively. Phlebotomy and Cell Saver (CS) were used following preestablished criteria.

Results: The mean number of intraoperative RBC units transfused was $0.3 \pm 0.7$. No plasma, nor platelets, nor cryoprecipitate were transfused. Seventy-nine percent of the patients received no blood products during their liver transplantation. The average final hemoglobin value was $84.8 \pm$ $18.3 \mathrm{~g} / \mathrm{l}$. In 28 patients (45.9\%), intraoperative phlebotomy and CS were used either together or separately. The average 24 and 72 hours creatinine postoperatively was the same as the preoperative one. There were neither cardiac complications nor any neurological complications. There was no intraoperative death nor any death in the 30-days period postoperatively. The oneyear survival rate was $91.4 \%$.

Conclusion: The avoidance of plasma transfusion and maintenance of a low CVP prior to the anhepatic phase were associated with a decrease in RBC transfusion during liver transplantation. Previous reports indicating that it is neither useful nor necessary to correct coagulation defects with plasma transfusion prior to liver transplantation are further corroborated by this prospective survey. We believe that this work also supports the practice of lowering CVP in order to reduce blood loss during liver dissection without any deleterious effect. 